\title{
Calcium-41 Chloride Aqueous Solution
}

National Cancer Institute

\section{Source}

National Cancer Institute. Calcium-41 Chloride Aqueous Solution. NCI Thesaurus. Code C85473.

An orally bioavailable aqueous solution containing the chloride salt of the radioisotope calcium-41 (41Ca) with phosphate-binding and radioisotopic activities. Upon administration of calcium- 41 chloride aqueous solution, calcium- 41 is preferentially taken up by osteoblasts, which generate mineralized osteoid containing calcium. Calcium-41 accumulation and turnover in bone can be measured with bone scintig raphy and urinary isotope excretion. 